\title{
Effect of matcha consumption on gut microbiota in healthy Japanese individuals: study protocol for a double-blind crossover interventional study
}

Maya Takegami ( $\sim$ choco314@koto.kpu-m.ac.jp )

Kyoto Prefectural University of Medicine: Kyoto Furitsu Ika Daigaku

Yoshitaka Hashimoto

Kyoto Prefectural University of Medicine: Kyoto Furitsu Ika Daigaku

Tomoki Miyoshi

Kyoto Prefectural University of Medicine: Kyoto Furitsu Ika Daigaku

Chihiro Munekawa

Kyoto Prefectural University of Medicine: Kyoto Furitsu Ika Daigaku

\section{Takashi Yoshimura}

Kyoto Prefectural University of Medicine: Kyoto Furitsu Ika Daigaku

Hanako Nakajima

Kyoto Prefectural University of Medicine: Kyoto Furitsu Ika Daigaku

Tomonori Kimura

Kyoto Prefectural University of Medicine: Kyoto Furitsu Ika Daigaku

Takuro Okamura

Kyoto Prefectural University of Medicine: Kyoto Furitsu Ika Daigaku

Naoko Nakanishi

Kyoto Prefectural University of Medicine: Kyoto Furitsu Ika Daigaku

Yuki Kitao

Kyoto-fu

Kazutsugu Kinoshita

Kyoto-fu

Masahide Hamaguchi

Kyoto Prefectural University of Medicine: Kyoto Furitsu Ika Daigaku

Michiaki Fukui

Kyoto Prefectural University of Medicine: Kyoto Furitsu Ika Daigaku

\section{Study Protocol}

Keywords: Matcha, dysbiosis, microbiota, double-blind crossover interventional study 
Posted Date: April 12th, 2021

DOl: https://doi.org/10.21203/rs.3.rs-391684/v1

License: (c) (i) This work is licensed under a Creative Commons Attribution 4.0 International License. Read Full License

Version of Record: A version of this preprint was published at Journal of Clinical Biochemistry and Nutrition on January 1st, 2021. See the published version at https://doi.org/10.3164/jcbn.21-110. 


\section{Abstract}

\section{Background:}

This study compares and clarifies the changes in intestinal flora resulting from the continuous consumption of two types of matcha.

\section{Methods:}

Healthy adults will consume two types of matcha tea for four weeks, and differences in the intestinal microflora before and after drinking will be compared. Gut microbiota will be identified using nextgeneration sequencing (NGS). Phylogenetic classification of the enterobacteria will be performed based on sequence similarities. The relative proportions of the classified enterobacteria to the total nucleotide sequences will be compared between the samples obtained from the two groups consuming different matcha.

\section{Discussion:}

The continuous consumption of matcha may improve dysbiosis and prevent atherosclerosis. The effects may vary according to the type of matcha used.

\section{Trial registration:}

The study was registered with university hospital medical information network (UMIN) (UMIN000040303), and all participants gave their written informed consent. Registered 1 November 2020, https://upload.umin.ac.jp/cgi-open-bin/ctr/ctr_view.cgi?recptno=R000045982.

\section{Introduction}

\section{Background}

Cardiovascular disease (CVD), including coronary artery disease, cerebral infarction, and peripheral artery disease, is one of the major causes of death in Japan (1). Not only is CVD a cause of death, but it also increases the social burden of both acute and post-acute care (2). Atherosclerosis is a major cause of CVD $(3,4)$. Thus, the prevention of atherosclerosis is important to prevent future CVD and related events.

In this decade, many studies have reported that the gut microbiota plays a pivotal role in human health and disease, including atherosclerosis. Regulating the balance of the gut microbiota may prevent the development of atherosclerosis (5). In fact, the mechanism by which hypertension, a risk factor for the development of atherosclerosis, occurs is through the disruption of gut microbiota (dysbiosis) caused by a high-salt diet (6).

Matcha has long been one of the most popular foods in Japanese food culture in a tea ceremony, and its health benefits have long been recognized. Unlike sencha, matcha contains high concentrations of 
polyamines, amino acids, such as theanine and glutamic acid, tea catechins, and polyphenols; additionally, it can be consumed as whole tea leaves (7). The polyamines present in matcha have antiaging effects (8). Moreover, matcha contains high levels of polyamines and, additionally, matcha increases the polyamine production of the gut microbiota (9). Therefore, matcha intake is expected to improve dysbiosis.

There are many different varieties of matcha. Polyamines, catechins, theanines, and other components in matcha vary depending on the variety. Possibly, the effects of matcha on the gut microbiota may vary depending on the variety. However, no previous study has clarified this point.

Our objectives are to investigate the effect of matcha consumption on the gut microbiota of healthy Japanese individuals using two kinds of tea leaves.

\section{Method}

This study will be conducted at the Department of Endocrinology and Metabolism, Kyoto Prefectural University of Medicine (Kyoto, Japan) and HP (htyearstps://kpum-1nai.jp/metabo/). Trial design is a double-blind crossover interventional study. The principal investigator or the person in charge will explain the study in a written form and obtain written consent from the participants themselves. Healthy adults (age 20 years or older on the date of consent, regardless of gender) who have been fully informed of the contents of this study and have given their consent are eligible. Healthy participants will be recruited through public recruitment. After that, the participants will be randomized by a simple randomization method to the high-quality matcha: Shitimeinomukashi, sold by Horiishichimeien (Uji, Japan, https://ujishichimeien.co.jp/) group and the processing matcha: no name sold by Kyoeiseicha Co., Ltd. (Osaka, Japan, https://www.kyoeiseicha.co.jp/company/index.html) group by a person in charge who is not involved in any other procedure of this study (Figure 1).

The following inclusion and exclusion criteria will be followed.

Participants who fulfil the following will be included:

(1) Participants who are able to drink at least one cup of matcha green tea daily

(2) Participants who have not received any antibiotics, undergone dietary interventions, and changed their oral medications in the last 3 months, and

(3) Participants between 20 and 80 years of age at the time of obtaining consent.

Participants who fall into any of the following categories will be excluded:

(1) Pregnant women, women who may be or intend to become pregnant, and women who are breastfeeding.

(2) Participants with a history of anemia or those undergoing treatment for it 
(3) Participants who have a confirmed diagnosis of or are under treatment for chronic diseases, such as diabetes, hyperlipidemia, and hypertension

(4) Participants with a history of cardiovascular disease, renal disease, liver disease, abnormal thyroid function, or malignancy, and

(5) Participants with diagnoses of or participants are who are undergoing treatment for allergic diseases, such as asthma or immune disorders

The following is a description of the intervention (Table 1 and Figure 1).

Visit 0: After obtaining consent from 20 healthy participants sing an explanatory document and filling out a registration form, 10 participants will be randomly assigned to the high-quality-matcha-first and processing-matcha-first groups.

Visit 1: After the registration, fasting blood and urine samples will be collected and the vascular endothelial function and dietary habits will be evaluated. At the same time, stool samples will be collected, and stool characteristics at the time of collection will be evaluated. The first day is set as the day after stool sample collection.

From the first day, $2 \mathrm{~g}$ of matcha dissolved in water or hot water is to be consumed every morning.

Visit 2: Visit 2 will be performed 28-35 days after Visit 1. Stool samples will be collected, and stool characteristics at the time of stool collection will be evaluated. Fasting blood and urine samples will be collected, and vascular endothelial function and dietary habits will be evaluated.

Visit 3: Visit 3 will be performed 28 days (21-35 days) after Visit 2. Fasting blood and urine samples will be collected, and vascular endothelial function and dietary habits will be evaluated. At the same time, stool samples will be collected, and stool characteristics at the time of stool collection will be evaluated. The first day is set as the day after stool sample collection. From the first day, $2 \mathrm{~g}$ of matcha, which is of the other type, dissolved in water or hot water is to be consumed every morning.

Visit 4: Visit 4 will be performed 28-35 days after Visit 3. Fasting blood and urine samples will be collected, and vascular endothelial function and dietary habits will be evaluated. During the study period, the participants will be advised to maintain the same dietary, drinking, smoking, and exercise habits as before;

\section{Data collection and management}

Samples of gut microbiota, blood, urine, and feces, will be obtained at each visit. In addition, vascular endothelial function will also be evaluated at each visit.

Gut microbiota. 
Fecal sample collection and the analyses of gut bacterial composition will be performed as previously described (10). Fecal samples will be collected in a guanidine thiocyanate solution (feces collection kit; Techno Suruga Lab, Shizuoka, Japan). Isolation of genomic DNA and analysis of DNA by 16S rRNA metagenomic sequencing will be performed by Bioengineering Lab. Co. Ltd. (Kanagawa, Japan). The amplicon sequence variant (ASV) table will be generated using Quantitative Insights Into Microbial Ecology 2 (QIIME2) (11). QIIME2 was developed on the basis of a plugin architecture that allows third parties to contribute functionality (https://library.qiime2.org). The prediction of the functional profiles from the 16S rRNA dataset will be performed using the Phylogenetic Investigation of Communities by Reconstruction of Unobserved States (PICRUSt2) software (http://picrust.github.com) (12). The 16S rRNA gene amplicons will be functionally annotated and mapped onto networks in the KEGG database (release 70.0), and functional profiles of the gut microbiota will be predicted.

Serum and urinary samples

The blood samples will be obtained after overnight fasting, and the levels of aspartate aminotransferase (AST), alanine aminotransferase (ALT), gamma-glutamyl transferase (GGT), total cholesterol, triglyceride (TG), high-density lipoprotein cholesterol (HDL-C), low-density lipoprotein cholesterol (LDL-C), highsensitivity C-reactive protein (hsCRP), fasting plasma glucose (FPG), and hemoglobin A1c (HbA1c) will be assessed.

Urinary samples from the second morning's urine will also be obtained and urine creatinine, sodium, chloride, and potassium levels will be evaluated. Proteinuria, hematuria, glucosuria, urinary ketone, urinary power of hydrogen $(\mathrm{pH})$, and urine specific gravity will be defined using a urine dipstick test.

Dietary assessment (survey of ingested foods and nutrients)

In this study, food and nutrient intake will be evaluated using a brief-type self-administered diet history questionnaire (BDHQ) (13) (14). The BDHQ has been sufficiently validated, enabling relatively simple surveying of the long-term dietary state; additionally, it is suitable for large-scale epidemiological studies.

Metabolomic and lipidemic analyses

Metabolomic and lipidemic analyses will be performed on serum, urine, and fecal samples. The samples will be derivatized using the online solid-phase derivatization system SPE-GC/MS (AiSTI SCIENCE Co., Ltd. Wakayama, Japan) or the fatty acid methylation kit (Nacalai Tesque, Inc. Kyoto, Japan) and analyzed using the GC/MS system (Agilent 7890 B +7000D, Agilent Technologies, Inc. Santa Clara, United States). Pathway analyses will be performed to identify changes in metabolites due to the consumption of high-quality matcha and processed matcha.

Vascular endothelial function

Peripheral arterial tonometry signals will be obtained using the EndoPAT 2000 device (Itamar Medical Inc., Caesarea, Israel) (15) (16). Endothelial function will be measured via the reactive hyperemia- 
peripheral arterial tonometry (RH-PAT) index (17).

\section{Statistical methods}

Primary outcome is that to investigate the difference in the changes in the gut microbiota between the high-quality and processing-matcha participant groups, the weighted average differences (WAD) method will be used (18). In this method, genera are ranked by comprehensively assessing higher expression, higher weights, and fold changes. The WAD method was found to be effective in transcriptome analysis. Secondary outcomes are to investigate the difference in the functional profiles of the gut microbiota between the high-quality and processing matcha participant groups, the WAD method will be used. In addition, changes in the metabolites of serum, urine, and fecal samples, dietary intake, fecal condition, and peripheral vascular endothelial function will also be evaluated by paired t-test or Wilcoxon signedrank test. Furthermore, multiple regression analyses will be carried out to check the correlation of gut microbiota with diet, stool properties, serum and urinary data, and vascular endothelial function.

\section{Discussion}

Dietary habits are known to be associated with atherosclerosis through changes in the gut microbiota. Through this study, we can clarify the effect of matcha on the improvement of gut microbiota. In addition, we will be able to clarify the effect of matcha on the improvement of vascular endothelial function. This study is a pilot study, and based on the results of this study, it is necessary to further elucidate the mechanism of matcha's effect on the body and to verify its effectiveness in preventing atherosclerosis.

\section{Abbreviations}

UMIN: university hospital medical information network

NGS: Next Generation Sequencer

BDHQ: brief-type self-administered diet history questionnaire

AST: aspartate aminotransferase

ALT: alanine aminotransferase

GGT: gamma-glutamyl transferase

TG: triglycerides

HDL-C: high-density lipoprotein, cholesterol

LDL-C: low-density lipoprotein cholesterol

hsCRP: high-sensitivity C-reactive protein 
$\mathrm{pH}$ : power of hydrogen

FPG: fasting plasma glucose

HbA1c: hemoglobin A1c

NGSP: National Glycohemoglobin Standardization Program

ASV: amplicon sequence variant

QIIME2: Quantitative Insights Into Microbial Ecology 2

PICRUSt2: Reconstruction of Unobserved States

PWA: pulse wave amplitude

RH-PAT : Reactive hyperemia-peripheral arterial tonometry

\section{Declarations}

\section{Ethics approval and consent to participate}

This study is registered at UMIN000040303. This study was approved by the Certified Review Board of Kyoto Prefectural University of Medicine. (ID: ERB-C-1818, September 28th, 2020)

\section{Acknowledgements}

We would like to thank Editage (www.editage.com) for English language editing.

\section{Author's Contributions}

MT is the Chief Investigator; he conceived the study, led the proposal and protocol development. TM contributed to study design and to development of the proposal. $\mathrm{MH}$ was the lead trial methodologist. All authors read and approved the final manuscript.

\section{Funding}

This study was funded by Tea Industry Research Division, Kyoto Prefectural Agriculture, Forestry and Fisheries Technology.

\section{Availability of data and material}

Data sharing is not applicable to this article as no datasets were generated or analysed during the current study.

\section{References}


1. Report of. Vital Statistics of Ministry of Health, Labour and Welfare.

2. https://. Accessed 19 Jan 2021.

3. Portal site of official statistics of Japan.

4. https://. Accessed 19 Jan 2021.

5. WHO Global Health Estimates. 2000-2015.

http://www.who.int/healthinfo/global_burden_disease/estimates/en/index1.html. Accessed 19 Jan 2021.

6. Emelia J, Benjamin MJ, Blaha SE, Chiuve M, Cushman SR, Das R, Deo, et al. Heart Disease and Stroke Statistics 2017 update: A report from the American Heart Association. Circulation. 2017;135:1-459.

7. Masafumi Takeda T, Yamashita N, Sasaki K, Nakajima T, Kita M, Shinohara, et al. Oral administration of an active form of vitamin D3 (calcitriol) decreases atherosclerosis in mice by inducing regulatory T cells and immature dendritic cells with tolerogenic functions. Arterioscler Thromb Vasc Biol. 2010;30:2495-503.

8. Faraco G, Brea D, Garcia-Bonilla L, Wang G, Racchumi G, Chang H, et al. Dietary salt promotes neurovascular and cognitive dysfunction through a gut-initiated TH17 response. Nat Neurosci. 2018;21:240-9.

9. Food composition database Ministry of Education. Culture, Sports, Science and Technology. https://fooddb.mext.go.jp/. Accessed 19 Jan 2021.

10. Soda K, Dobashi Y, Kano Y, Tsujinaka S, Konishi F. Polyamine-rich food decreases age-associated pathology and mortality in aged mice. Exp Gerontol. 2009;44:727-32.

11. Kibe R, Kurihara S, Sakai Y, Suzuki H, Ooga T, Sawaki E, et al. Upregulation of colonic luminal polyamines produced by intestinal microbiota delays senescence in mice. Sci Rep. 2014;4:1-11.

12. Inoue $R$, Ohue-Kitano R, Tsukahara T, Tanaka M, Masuda S, Inoue T, Yamakage H, Kusakabe T, Hasegawa K, Shimatsu A, Satoh-Asahara N. Prediction of functional profiles of gut microbiota from $16 \mathrm{~S}$ rRNA metagenomic data provides a more robust evaluation of gut dysbiosis occurring in Japanese type 2 diabetic patients. J Clin Biochem Nutr. 2017 Nov;61(3):217-21.

13. Caporaso JG, Kuczynski J, Stombaugh J, Bittinger K, Bushman FD, Costello EK, Fierer N, Peña AG, Goodrich JK, Gordon JI, Huttley GA, Kelley ST, Knights D, Koenig JE, Ley RE, Lozupone CA, McDonald D, Muegge BD, Pirrung M, Reeder J, Sevinsky JR, Turnbaugh PJ, Walters WA, Widmann J, Yatsunenko T, Zaneveld J, Knight R. QIIME allows analysis of high-throughput community sequencing data. Nat Methods. 2010 May;7(5):335-6.

14. Langille MG, Zaneveld J, Caporaso JG, McDonald D, Knights D, Reyes JA, Clemente JC, Burkepile DE, Vega Thurber RL, Knight R, Beiko RG, Huttenhower C. Predictive functional profiling of microbial communities using 16S rRNA marker gene sequences. Nat Biotechnol. 2013 Sep;31(9):814-21.

15. Satomi Kobayashi K, Murakami S, Sasaki H, Okubo N, Hirota A, Notsu, et al. Comparison of relative validity of food group intakes estimated by comprehensive and brief-type self-administered diet 
history questionnaires against $16 \mathrm{~d}$ dietary records in Japanese adults. Public Health Nutr. 2011;14:1200-11. 5).

16. Satomi Kobayashi 1. Honda S, Murakami K, Sasaki S, Okubo H, Hirota N, et al. Both comprehensive and brief self-administered diet history questionnaires satisfactorily rank nutrient intakes in Japanese adults. J Epidemiol. 2012; 22:151-9.

17. Bonetti PO, Pumper GM, Higano ST, Holmes DR, Kuvin JT, Lerman A. Noninvasive identification of patients with early coronary atherosclerosis by assessment of digital reactive hyperemia. J Am Coll Cardiol. 2004;44:2137-41.

18. Hamburg NM, Keyes MJ, Larson MG, Vasan RS, Schnabel R, Pryde MM, et al. Cross-sectional relations of digital vascular function to cardiovascular risk factors in the Framingham Heart Study. Circulation. 2008;117:2467-74.

19. Bonetti PO, Barsness GW, Keelan PC, Schnell TI, Pumper GM, Kuvin JT, et al. Enhanced external counterpulsation improves endothelial function in patients with symptomatic coronary artery disease. J Am Coll Cardiol. 2003;41:1761-8.

20. Kadota K, Nakai Y, Shimizu K. A weighted average difference method for detecting differentially expressed genes from microarray data. Algorithms Mol Biol. 2008;3:8.

\section{Table}

Table 1: Timeline of the participants

\begin{tabular}{|c|c|c|c|c|c|}
\hline \multicolumn{1}{|c|}{ Schedule } & Visit 0 & Visit 1 & Visit 2 & Visit 3 & Visit 4 \\
\hline Obtaining consent & $\bullet$ & & & & \\
\hline $\begin{array}{l}\text { Blood test, } \\
\text { urine test }\end{array}$ & & $\bullet$ & $\bullet$ & $\bullet$ & $\bullet$ \\
\hline RH-PAT & & $\bullet$ & $\bullet$ & $\bullet$ & $\bullet$ \\
\hline Stool test & & $\bullet$ & $\bullet$ & $\bullet$ & $\bullet$ \\
\hline
\end{tabular}

口: perform; RH-PAT: Reactive hyperemia-peripheral arterial tonometry.

\section{Figures}




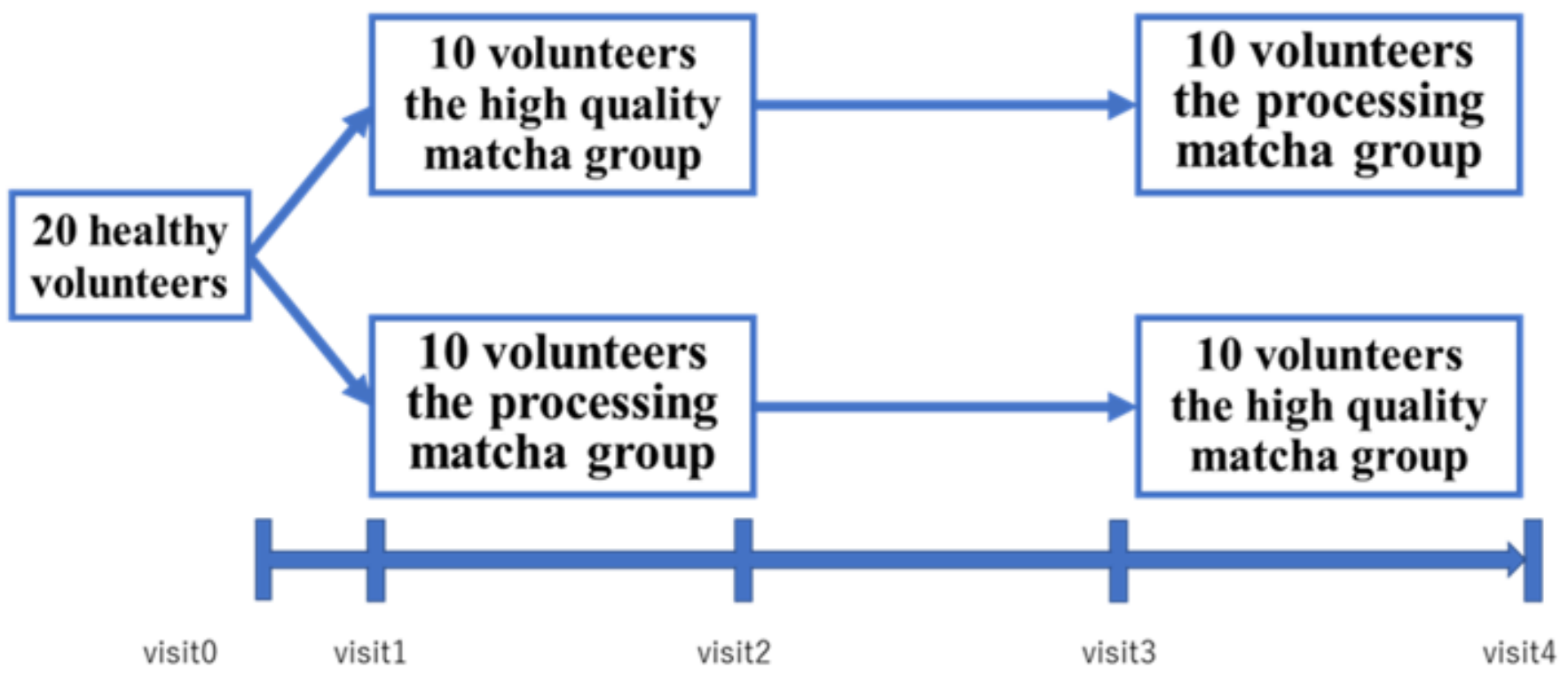

Figure 1

The registration of volunteers. 